\title{
The investigation of dynamic behaviour of a structure using wave-based substructuring method
}

\author{
Ahmad Burhani Ahmad Basri ${ }^{1}$, Muhamad Norhisham Abdul Rani ${ }^{1,{ }^{*}, \text { Mohd Azmi Yunus }}{ }^{1}$, \\ Liyana Roslan ${ }^{2}$, and Wan Imaan Izhan Wan Iskandar Mirza ${ }^{1}$ \\ ${ }^{1}$ Center of Excellence of Dynamic and Control, Faculty of Mechanical Engineering, Universiti \\ Teknologi MARA Kampus Shah Alam, 40450 Shah Alam, Selangor, Malaysia \\ ${ }^{2}$ Faculty of Mechanical Engineering, Universiti Teknologi MARA Kampus Pasir Gudang, 81750 \\ Masai, Johor, Malaysia
}

\begin{abstract}
There is an increasing need for accurate, efficient and economical methods for the investigation of the dynamic behaviour of large complex structures within the engineering community. The component mode synthesis (CMS) has been perceived by the community to be an attractive efficient method for the investigation. However, the method has substantial shortcomings, particularly in analysing a structure having a large number of interface degrees of freedom (DOFs) between substructures. This paper puts forward a method, based upon the wavebased substructuring (WBS) for the investigation of the dynamic behaviour of a structure with a large number of interface DOFs. The finite element method is used to construct the full finite element model of the structure and NASTRAN 103 is used for the normal modes analysis. A new finite element model of the structure with reduced interface DOFs is constructed based on the WBS. The measurement of the dynamic behaviour of the structure is carried out using free-free boundary conditions and an impact hammer test. The predicted results of the proposed method are then compared with those from the full finite element model and experimental counterparts. The accuracy and efficiency of the proposed method are discussed and illustrated with two different case studies.
\end{abstract}

\section{Introduction}

Over time, engineering structure has been developed to a certain level of large numerical model which possesses thousand numbers of DOFs. Deriving the mathematical model, especially for a complex structure that is having numbers of substructures, boundary conditions and enormous number of DOFs is a very challenging and difficult task even for the modern computer. Despite the presence of the modern computer that has been used by most of the industrial companies in increasing the work efficiency, it seems to be unable to

* Corresponding author: mnarani@salam.uitm.edu.my 
perform the expected level of efficiency in terms of the iteration time. The application of conventional finite element analysis (FEA) which uses a full finite element model has been widely used and the effort of computing the mathematical model seems to be unsuitable when the computational is too expensive due to the repeated iteration solution performed.

To overcome this problem, a technique called component mode synthesis (CMS) has been introduced to enhance the computational time process. It is believed to be a very practical and efficient method for investigating the structural normal modes. The method has been widely used in the engineering field for solving complex high profile engineering structural field. In the automotive world, CMS method is used in increasing the speed of vehicle development and system-level performance assessment[1]. Not only that, in civil engineering, the method is used in the study of the bridge frame and the capability of the method towards model updating scheme[2]. A rapid analysis of the space shuttle which is used in aerospace industries has shown the success of the method in predicting the dynamic behaviour of the structure[3]. Based on the case stated, it has clearly shown the capability of the rapid CMS method in analysing the complex engineering structure.

Unfortunately, since the needs of the FEM is highly demanded by the industry and application of the method is not limited to only a few kind of structures, computing the complex engineering structure through CMS method seems to be impractical. This problem arises when the substructures are coupled with a very large interface nodes[4]. Since the efficiency of this method highly depends on the boundary nodes in which for certain structures that are still having a very large interface nodes between the substructures, the method is unable to speed up the computational time[5,6]. Even worse, for a structure that has a limited number of the substructure, there is no other choice but only to mandatory select the large interface as the interface representation [7]. This situation has brought difficulty in using the CMS method as the reduced order scheme. Although much work has been done to date, more studies need to be conducted to find out the most reliable method that can be effectively and accurately used without highly depending on the full interface nodes.

In this paper, the work is concerned with the use of wave-based substructuring method as the reduced scheme in investigating the dynamic behaviour of a structure which is perceived able to analyse a large number of interface DOFs. It is a method, in which instead of all the interface nodes are used to be analysed as practised in CMS method, that presents a substructuring approach where the interface is written as a combination of a set of basis deformation called waves [5]. A smaller number of DOFs is used to represent the interface representation that gives a better result in terms of the computational time. As the number of waves (interface nodes) is much lower than the interface DOFs, a rapid prediction can be obtained compared to the conventional CMS method [8,9]. Therefore, as the interface representation is smaller, easier reduction procedure and shorter time frame of the iteration process can be obtained while the accuracy of the prediction is preserved.

\section{Theory of wave-based substructuring}

Consider an undamped structure with no external forces applied to it, the FE matrix form will be:

$$
\mathbf{M} \ddot{\mathbf{x}}+\mathbf{K} \ddot{\mathbf{x}}=0
$$

The FE structure is divided into several non-overlapping substructures to obtain an assembled system. For each substructure, the DOFs, $\mathrm{x}$ are separated into interior DOFs, $\mathrm{x}_{\mathrm{i}}$ and boundary DOFs or junction DOFs, $x_{j}$ at the boundary [10]. The system matrices for each substructure will be: 


$$
\left[\begin{array}{ll}
\mathbf{M}_{\mathrm{ii}} & \mathbf{M}_{\mathrm{ij}} \\
\mathbf{M}_{\mathrm{ji}} & \mathbf{M}_{\mathrm{jj}}
\end{array}\right]\left[\begin{array}{c}
\ddot{\mathrm{X}}_{\mathrm{i}} \\
\ddot{\mathrm{x}}_{\mathrm{j}}
\end{array}\right]+\left[\begin{array}{ll}
\mathbf{K}_{\mathrm{ii}} & \mathbf{K}_{\mathrm{ij}} \\
\mathbf{K}_{\mathrm{ji}} & \mathbf{K}_{\mathrm{jj}}
\end{array}\right]\left[\begin{array}{c}
\mathrm{X}_{\mathrm{i}} \\
\mathrm{x}_{\mathrm{j}}
\end{array}\right]=\left[\begin{array}{c}
\mathrm{f}_{\mathrm{i}} \\
\mathrm{f}_{\mathrm{j}}
\end{array}\right]
$$

In the WBS approach, the method will limit the junction DOFs, $x_{j}$ by reducing the interface description by introducing them to a set of basis function $\mathrm{W}$, weighted with involvement factor $\mathrm{p}$ :

$$
\mathrm{x}_{\mathrm{j}}=W \times p
$$

The Equation (3) is substituted into Equation (2):

$$
\left[\begin{array}{cc}
\mathbf{M}_{\mathrm{ii}} & \mathbf{M}_{\mathrm{ij}} \mathrm{W} \\
\mathrm{W}^{\mathrm{T}} \mathbf{M}_{\mathrm{ji}} & \mathrm{W}^{\mathrm{T}} \mathbf{M}_{\mathrm{jj}} \mathrm{W}
\end{array}\right]\left[\begin{array}{c}
\ddot{\mathrm{x}}_{\mathrm{i}} \\
\ddot{\mathrm{p}}
\end{array}\right]+\left[\begin{array}{cc}
\mathbf{K}_{\mathrm{ii}} & \mathbf{K}_{\mathrm{ij}} \mathrm{W} \\
\mathrm{W}^{\mathrm{T}} \mathbf{K}_{\mathrm{ji}} & \mathrm{W}^{\mathrm{T}} \mathbf{K}_{\mathrm{jj}} \mathrm{W}
\end{array}\right]\left[\begin{array}{c}
\mathrm{x}_{\mathrm{i}} \\
\mathrm{p}
\end{array}\right]=\left[\begin{array}{c}
\mathrm{f}_{\mathrm{i}} \\
\mathrm{V}^{\mathrm{T}} \mathrm{f}_{\mathrm{j}}
\end{array}\right]
$$

At this point, the set of basis functions is known as "waves". As the number of waves is lower than the junction DOFs, $\mathrm{x}_{\mathrm{j}}$, this substitution will reduce the size of the interface FE matrix equation.

Continuity and equilibrium condition must be translated into interface basis functions to perform the assembly for the substructures. For a rigid connection[4] between $a$ and $b$ substructures, the continuity and equilibrium conditions are as follow:

$$
x_{j}^{(a)}=x_{j}^{(b)} \text { and } f_{j}^{(a)}=f_{j}^{(b)}
$$

In WBS, the assembly of two substructures, $a$ and $b$ will have the junction DOFs expressed as $x_{j}^{(a)}=W^{(a)} \times p^{(a)}, x_{j}^{(b)}=W^{(b)} \times p^{(b)}$, respectively. The same condition is applied to the involvement factor $\mathrm{p}$ :

$$
p^{(a)}=p^{(b)} \text { and } f_{j}^{(a)}=f_{j}^{(b)}
$$

\section{Academic case}

In this section, there are two cases that have been investigated in order to assess the efficiency and the accuracy of the proposed method namely a normal size two-plate assembly case and 15 times bigger two-plate assembly case. The efficiency of the WBS method in computing the iteration was validated by comparing it with the full FE analysis and the conventional Craig-Bampton method and in terms of the accuracy, it was validated with the experimental counterparts. For the FE solver, MSC. Nastran [11] was used and LMS Virtual.Lab [12] as the pre- and post-processing. MATLAB [13] was used to validate the correlation of the result with the experimental result.

\subsection{Case study 1: Normal size two-plate assembly}

The model under the investigation is a large flat span structure that mimics a simplified aeroplane shape. The assembly structure consists of two substructures, Part A and Part B as shown in Figure 1. The model meshed into $10 \mathrm{~mm}$ meshing size for both parts. 


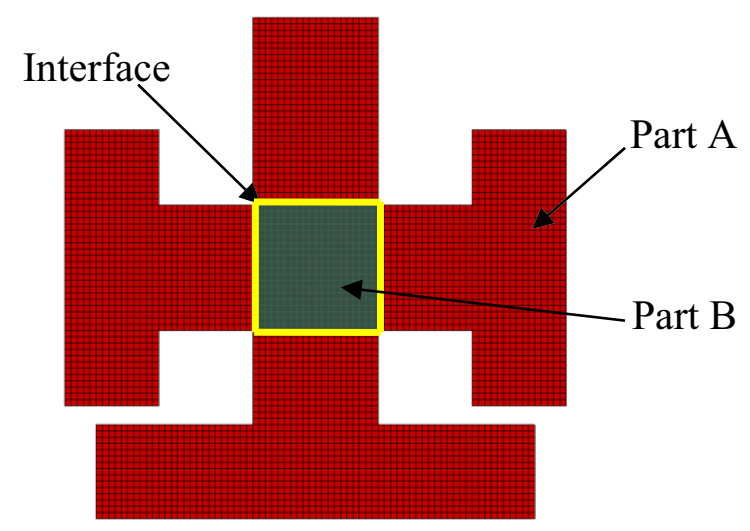

Fig. 1. Normal size structure consists of two-plate assembly (Part A:Red, Part B:Green).

The structure is a $4 \mathrm{~mm}$-thick aluminium made and $0.8 \mathrm{~m}$ by $0.8 \mathrm{~m}$ size which has the following properties:

- $\quad$ Young's Modulus, $\mathrm{E}=73.58 \mathrm{GPa}$

- Poisson Coefficient, $\mathrm{n}=0.3$

- Mass Density, $\mathrm{r}=2877 \mathrm{~kg} / \mathrm{m}^{3}$

- Part A consists of 4168 nodes and 3870 quad elements.

- Part B consists of 441 nodes and 400 quad elements.

In between the two substructures, the interface nodes which consist of 80 coincident nodes were connected by rigid connections as shown in Figure 1(yellow line). Normal modes analysis was performed to obtain the first 16 normal modes. All the calculation was performed on an Intel(R) Core(TM) i7-3610QM CPU (2.30GHz, 8GB RAM) running on Windows 7 operating system, using MSC. Nastran v2012.

\subsection{Case study 2: 15 times bigger two-plate assembly}

The model under investigation is a large flat span structure that mimics a simplified aeroplane shape. The assembly structure consists of two substructures, Part A and Part B in which was increased in size to 15 times bigger than the normal structure size used in Case Study 1 . The purpose of increasing the size of the structure was to increase the number of nodes and elements so that a bigger difference in terms of the computational time between full FE, WBS method and Craig-Bampton method can be obtained. The model meshed into $10 \mathrm{~mm}$ meshing size for both parts. The same material properties were used in this case study. The geometry specifications of the structure are as follows:

- $\quad$ Part A consists of 875220 nodes and 870750 quad elements.

- Part B consists of 90601 nodes and 90000 quad elements.

In between the two substructures, the interface nodes which are consist of 1200 coincident nodes was connected by the rigid connection. Normal modes analysis was performed to obtain the first 16 normal modes. All the calculation was performed on an Intel(R) Core(TM) i7-3610QM CPU (2.30GHz, 8GB RAM) running a Windows 7 operating system, using MSC. Nastran v2012. 


\section{Results and discussion}

In this study, the natural frequencies and mode shapes are the interested modal parameters to be observed which were obtained from the finite element method and experimental modal analysis. The results of modal parameters and CPU time was recorded and tabulated in Table 1. Meanwhile, two reduced order method (Craig-Bampton and WBS) were used to study the capabilities of the methods in term of efficiency computing the iteration process. The results were tabulated in Table 3 .

For the first case study, all the normal mode analysis is performed in the frequency of interest range $0-120 \mathrm{~Hz} .25$ number of waves are used as the interface node which has been selected from the sensitivity analysis done (see Figure 2). In order to obtain an accurate and efficient results, one should aims at using the least number of waves that is enough to represent the substructure interface deformation analogous to the full FE model normal modes behaviour of the complete structure[14].

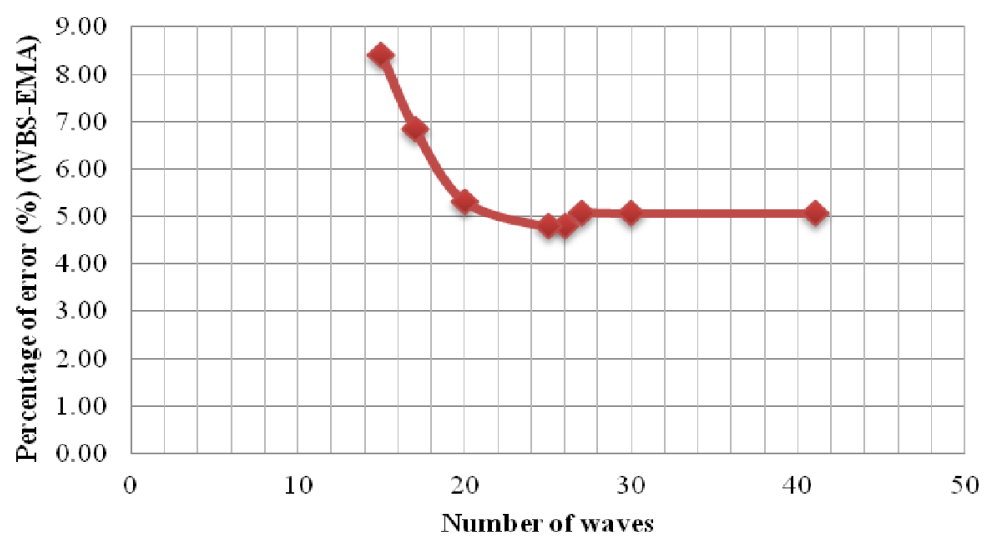

Fig. 2. Sensitivity analysis of waves. Comparison between the WBS-connected models and the EMA in terms of eigenfrequency for a different number of waves.

There are 10 elastic modes for the frequency of interest up till $120 \mathrm{~Hz}$. Since the number of DOFs is small in this model, the difference between the full FE and WBS method in terms of the computational time is not that significant. However, in the case of the accuracy of the method, a clear significant result is obtained. Two types of comparisons of results are shown in Table 1.

The first comparison is between the results of full FE and the test data. The comparisons of the results are tabulated in the column (IV). An error of $5.09 \%$ is obtained for this comparison. The second comparison is between the WBS model and the test data. The results of the comparisons are shown in Column (V) in Table 1. A lower percentage of error with a value of $4.79 \%$ is obtained. In terms of the MAC analysis, the average MAC value is 0.92 which indicates that the results have a very good agreement in terms of the mode shapes between the numerical and experimental work (see also Table 2).

It was found that the method not recommended to be used for a small structure since setting the reduction process for the substructure might take some time. It can be said that the method used was able to accurately maintain and significantly improved the level of accuracy. This indicates that WBS offers a benefit for reduction where the accuracy of the prediction is maintained even a smaller number of DOFs is used to analyse the structure. In order to get a better view on the efficiency of the WBS method, the second case study was performed. 
Table 1. Comparisons of natural frequencies obtained from the full FEM, WBS and EMA for the normal size structure.

\begin{tabular}{|c|c|c|c|c|c|c|}
\hline \multirow{2}{*}{ Mode } & (I) & (II) & & (III) & (IV) & (V) \\
\cline { 2 - 7 } & $\begin{array}{c}\text { Full FE } \\
\text { (Hz) }\end{array}$ & $\begin{array}{c}\text { WBS } \\
\text { (Hz) }\end{array}$ & MAC & $\begin{array}{c}\text { EMA } \\
(\mathbf{H z})\end{array}$ & $\begin{array}{c}\text { Error } \\
\text { between I } \\
\text { \& III (\%) }\end{array}$ & $\begin{array}{c}\text { Error } \\
\text { between II } \\
\text { \& III (\%) }\end{array}$ \\
\hline 1 & 16.38 & 16.38 & 0.9 & 16.27 & 0.67 & 0.67 \\
\hline 2 & 17.05 & 17.09 & 1.0 & 17.15 & 0.57 & 0.34 \\
\hline 3 & 31.25 & 31.25 & 1.0 & 31.29 & 0.14 & 0.12 \\
\hline 4 & 31.77 & 31.78 & 0.8 & 31.52 & 0.79 & 0.82 \\
\hline 5 & 37.33 & 37.35 & 1.0 & 37.87 & 1.43 & 1.39 \\
\hline 6 & 46.71 & 46.71 & 0.8 & 46.77 & 0.12 & 0.12 \\
\hline 7 & 73.82 & 73.83 & 1.0 & 74.23 & 0.56 & 0.54 \\
\hline 8 & 90.21 & 90.23 & 1.0 & 90.56 & 0.39 & 0.36 \\
\hline 9 & 110.41 & 110.43 & 1.0 & 110.17 & 0.22 & 0.23 \\
\hline 10 & 118.88 & 118.88 & 0.7 & 118.65 & 0.20 & 0.20 \\
\hline $\begin{array}{c}\text { CPU } \\
\text { Time (s) }\end{array}$ & 2 & 1 & & $\begin{array}{c}\text { Total } \\
\text { Error }\end{array}$ & 5.09 & 4.79 \\
\hline
\end{tabular}

Table 2. Comparison between the measured and predicted mode shapes of the structure.

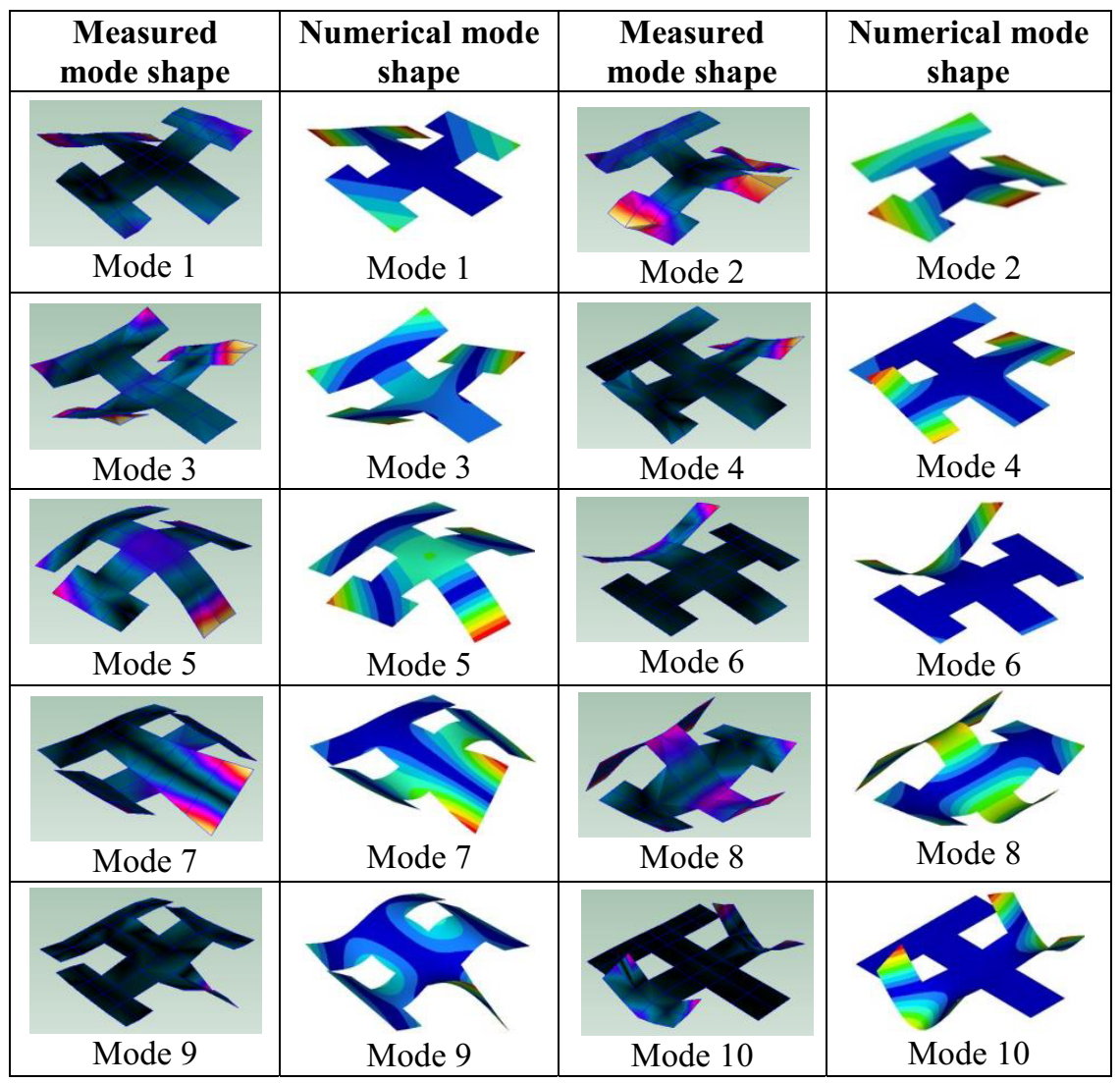


For the second case study, the structure has increased the size up to 15 times than the normal size as in Case Study 1. A total of 16 number of normal modes are computed. The results shown in Table 3 consist of three comparisons between the full FE, WBS and CraigBampton method.

Table 3. Comparisons between Full FE results and WBS results when the structure size was 15 times. bigger.

\begin{tabular}{|l|c|c|c|}
\hline & Full FE & Craig-Bampton & WBS \\
\hline Number of component normal modes & 16 & 16 & 16 \\
\hline Number of Nodes & 964621 & 1200 & 36 \\
\hline Reduction iteration time (s) & & 8907 & 1688 \\
\hline Assembly iteration time (s) & & 207 & 123 \\
\hline Total CPU Time (s) & 11349 & 9114 & 1811 \\
\hline Time reduction (\%) & & 19.7 & 84 \\
\hline
\end{tabular}

In full FE analysis, there are 964621 numbers of nodes which took 8585 seconds computational time for the analysis to complete. This situation shows the need to use an efficient method to calculate the iterative solution since it is taking too much time.

As the Craig-Bampton method was applied, instead of using all the nodes to perform the analysis, 1200 interface nodes are used to analyse the structure. It is found that the computational time has been reduced to 9114 seconds which indicated $19.7 \%$ time reduction from the full $\mathrm{FE}$ analysis. This result shows a huge lacking in efficiency still exists in the CPU time especially during the reduction iteration time. In addition, to able the reduction of the part A become achievable, the substructure (Part A) has to be decomposed into several new substructures. Part A was decomposed into four new substructures and analyse them separately. This situation has brought a new burden in reduction process since it will take more time to prepare a new substructure for an analysis.

As the WBS method is applied, the desired efficiency is achieved, as shown in Table 3. The notable achievement is gauged via the reduction in computational time between the full FE, Craig-Bampton and WBS analysis. Only 36 number of nodes is used to analyse the structure which gave the necessary time to compute the structure just by only 1811 seconds. This result clearly shows a significant reduction in the expenditure time by $84 \%$ time reduction from the full $\mathrm{FE}$ and 5 times quicker than the Craig-Bampton method. Plus, during the reduction process, it takes lesser time compared to Craig-Bampton method and a single substructure (Part A) was reduced without having to decompose it into several new substructures. Through the results obtained, a strong evident indicates that the WBS model is successfully, efficiently and accurately used to analyse a structural normal mode in comparison with the full FE model and Craig-Bampton model.

\section{Conclusions}

A wave-based substructuring approach for investigating the dynamic behaviour of a structure is presented. The efficiency and accuracy of WBS have been demonstrated in two different case studies. It has been shown that the WBS has accurately and efficiently predicted the modal parameters of the structure over the full FE and Craig-Bampton method. The significant advantages of the WBS method are that it is able to perform the investigation of the dynamic behaviour of the structure in the most efficient way. It is suggested that the proposed method is used when predicting a complex structure with a large number of iteration required.

A similar degree of accuracy can be obtained through the proposed method as obtained from the full FE model. Even the structure is analysed through the reduction model in 
WBS, the accuracy of the predicted is maintained as the same, while a rapid iteration process is used. This situation is proven that the WBS has the same capability as the full FE model in accurately predict the dynamic behaviour of a structure.

Authors gratefully acknowledge the Malaysia Ministry of Higher Education (MOHE) and Research Management Institute (RMI) of University Teknologi MARA (UiTM) for providing financial support for this study through the research acculturation grant scheme (RAGS)- 600-RMI/RAGS 5/3 (21/2015). Authors would also like to express their appreciation for helpful comments, suggestions and technical support given by Mr David Starbuck and Mr Mohamad Azwan Abdul Khair.

\section{References}

1. M. Bennur, "Superelement, Component Mode Synthesis, and Automated Multilevel Substructuring for Rapid Vehicle Development," SAE Int. J. Passeng. Cars - Mech. Syst., vol. 1, no. 1, pp. 2008-01-0287, (2008).

2. S. Weng, Y. Xia, Y.-L. Xu, H.-P. Zhu, "Substructure based approach to finite element model updating," Comput. Struct., vol. 89, no. 9-10, pp. 772-782, (2011).

3. R. R. J. Craig and C. J. Chang, "Substructure Coupling for Dynamic Analysis and Testing," (1977).

4. S. Donders, B. Pluymers, P. Ragnarsson, R. Hadjit, W. Desmet, "The wave-based substructuring approach for the efficient description of interface dynamics in substructuring," J. Sound Vib., vol. 329, no. 8, pp. 1062-1080, (2010).

5. A. Maressa, D. Mundo, S. Donders, W. Desmet, "A wave-based substructuring approach for concept modeling of vehicle joints," Comput. Struct., vol. 89, no. 23-24, pp. 2369-2376, (2011).

6. S. Donders, R. Hadjit, M. Brughmans, L. Hermans, W. Desmet, "A wave-based substructuring approach for fast vehicle body optimisation," Int. J. Veh. Des., vol. 43, no. $1 / 2 / 3 / 4$, p. 100, (2007).

7. E. Balmès, "Use of Generalized Interface Degrees of Freedom in Component Mode Synthesis," Proc. 14th Int. Modal Anal. Conf., pp. 1-7, (1996).

8. J. M. Mencik, D. Duhamel, "A wave-based model reduction technique for the description of the dynamic behavior of periodic structures involving arbitrary-shaped substructures and large-sized finite element models," Finite Elem. Anal. Des., vol. 101, pp. 1-14, (2015).

9. M. A. Tournour, N. Atalla, O. Chiello, F. Sgard, "Validation, performance, convergence and application of free interface component mode synthesis," Comput. Struct., vol. 79, no. 20-21, pp. 1861-1876, (2001).

10. S. Donders, R. Hadjit, M. Brughmans, L. Hermans, W. Desmet, "Speeding up vibroacoustic modification predictions using a Wave-Based Substructuring approach and ATV technology," Forum Acusticum Budapest 2005 4th Eur. Congr. Acustics, pp. 161-166, (2005).

11. "MSC. MSC/Nastran 2012." (2012).

12. "LMS International. LMS Virtual.Lab 13.5." (2015).

13. "The MathWorks Inc. MATLAB." (2013).

14. S. Donders, "Computer Aided Engineering Methodologies For Robust Automotive NVH Design,” Katholieke Universiteit Leuven, (2008). 